\title{
Energy Efficient Power Allocation for Heterogeneous Cloud Radio Access Network with Partial CSI
}

\author{
Jiakuo Zuo ${ }^{1+}$ and Chao Zuo ${ }^{2}$ \\ ${ }^{1}$ College of Internet of Things, Nanjing University of Posts and Telecommunications, Nanjing 210023, \\ China \\ ${ }^{2}$ College of Automation, Nanjing University of Aeronautics and Astronautics, Nanjing 210023, China
}

\begin{abstract}
In this paper, we consider power allocation for heterogeneous cloud radio access network (HCRAN). All the channels in HC-RAN are assumed to be block fading and only the statistical information of these channels can be acquired by the based band unit (BBU) instead of perfect channel state information (CSI). The power of the users is optimized via maximization of the averaged energy efficiency (EE) of HCRAN, under outage probability constraints and average transmit power constraint. First, the original nonconvex optimization problem is transformed into an equivalent optimization problem in subtractive form. Then, an efficient two loop iterative power allocation scheme is proposed. Simulation results demonstrate the improvements in terms of EE by using the proposed power allocation scheme compared with the traditional ergodic rate maximization algorithm.
\end{abstract}

Keywords: power allocation, energy efficient, heterogeneous cloud radio access network, fractional programming.

\section{Introduction}

In recent years, heterogeneous cloud wireless access network (HC-RAN) has attracted great research attention. HC-RAN can bring numerous benefits including low cost, flexible deployment of network and high utilization of resources[1]. Therefore, HC-RAN is considered one of the most promising solutions to address the key challenges in future wireless network [2].

Resource allocation is one of the key technologies for HC-RAN. In [3], a new economical spectral efficiency was defined to measure the cost of HC-RAN, and proposed a two loops algorithm to optimize the resource of HC-RAN. [4] studied joint user admission, association and power allocation problems for HCRAN, the above problem was modeled as a mixed integers nonlinear problems, then an outer approximation approach based on linearization method was proposed. [5] studied power allocation problem for nonorthogonal multiple access (NOMA) HC-RAN, and proposed an energy efficient power allocation scheme to allocate the power for different types base station (BS). The multiplexing gain of virtual base station pooling based on multi-dimensional Markov mode was researched in [6], and recursive formula and closed form approximation for the blocking probability were also derived. In [7], energy efficient resource block assignment and power allocation were studied for OFDMA-based HC-RAN. To maximize the energy efficiency performance, an iterative algorithm was proposed to achieve the global optimal solution. To maximize the average throughput and maintain the network stability, [8] studied the joint congestion control and resource optimization problem and an energy efficiency algorithm was proposed to balance throughput and delay. [9] studied joint power allocation, relay selection and networking selection for relay HC-RAN, and proposed an efficient algorithm based on relaxation method and nonlinear fractional programming.

\footnotetext{
${ }^{+}$Corresponding author.

E-mail address: zuojiakuo@njupt.edu.cn.
} 
However, all the above researches assume that HC-RAN can acquire perfect channel sate information (CSI). However, obtaining accurate estimation of CSI is challenging or even impossible. In this paper, we formulate the power allocation problem for energy efficient HC-RAN with Partial CSI as a nonconvex optimization problem. An efficient iterative power allocation scheme is proposed to solve the above problem.

\section{System Model and Proposed Formulation}

We consider an uplink resource sharing for HC-RAN with one macro cellular user (MU), one RRH user (RU). The MU and RU are served by MBS and RRH respectively. The MBS and RRH are connected to the Based Band Unit (BBU) via fronthual link. The RU reuses the same channel allocated to the MU. Denote the channel power gains (CPG) from MU to MBS by $g_{\mathrm{MU}}$, the CPG from RU to RRH by $\mathrm{g}_{\mathrm{RU}}$, the CPG from RU to the MBS by $h_{\mathrm{RM}}$, the CPG from the MU to the RRH by $\mathrm{h}_{\mathrm{MR}}$, respectively. Assume these channel power gains are ergodic over transmission blocks, and are independent, identically distributed. All these channels' statistical information is known at the BBU pool.

Let $v=\left[g_{\mathrm{MU}} h_{\mathrm{RM}} g_{\mathrm{RU}} h_{\mathrm{MR}}\right]$ denotes the power gain vector, $p_{\mathrm{MU}}$ and $p_{v}$ denote the transmit powers of MU and RU. Then, the rate of MU and RU in one fading channel state with channel realization $v$ are, respectively:

$$
\begin{gathered}
R_{\mathrm{MU}}=\log _{2}\left(1+\frac{p_{\mathrm{MU}} g_{\mathrm{MU}}}{N_{0}+p_{v} h_{\mathrm{RM}}}\right) \\
R_{\mathrm{RU}}=\log _{2}\left(1+\frac{p_{v} g_{\mathrm{RU}}}{N_{0}+p_{\mathrm{MU}} h_{\mathrm{MR}}}\right)
\end{gathered}
$$

where $N_{0}$ is the noise power.

Outage probability constraints of MU link and RU link are repressed as:

$$
\begin{aligned}
& \mathcal{P}\left(\log _{2}\left(1+\frac{p_{\mathrm{MU}} g_{\mathrm{MU}}}{N_{0}+p_{\nu} h_{\mathrm{RM}}}\right)<R_{\mathrm{min}}^{\mathrm{MU}}\right) \leq \sigma_{\mathrm{MU}} \\
& \mathcal{P}\left(\log _{2}\left(1+\frac{p_{\nu} g_{\mathrm{RU}}}{N_{0}+p_{\mathrm{MU}} h_{\mathrm{MR}}}\right)<R_{\mathrm{min}}^{\mathrm{RU}}\right) \leq \sigma_{\mathrm{RU}}
\end{aligned}
$$

where $\sigma_{\mathrm{RU}}$ and $\sigma_{\mathrm{MU}}$ are the maximum outage probability of RU and MU, respectively

The average transmit power constraint of RU link over all fading channel states is expressed as:

$$
\mathcal{E}\left[p_{v}\right] \leq P_{\max }
$$

where $\mathcal{E}$ denotes the expectation, $P_{\max }$ is the average transmit power threshold of RU.

Let $\xi_{\mathrm{EE}}$ denote the energy efficiency of the RU link averaged over all the fading states, and it can be defined as follows:

$$
\xi_{\mathrm{EE}}=\frac{\mathcal{E}\left\lfloor\log _{2}\left(1+\frac{p_{v} g_{\mathrm{RU}}}{N_{0}+p_{\mathrm{MU}} h_{\mathrm{MR}}}\right)\right\rfloor}{\mathcal{E}\left[p_{v}\right]+P_{\mathrm{c}}}
$$

where $P_{\mathrm{c}}$ denote the circuit power consumption of the RU.

Mathematically, the EE optimization problem can be written as:

$$
\begin{aligned}
& \text { OP1 } \max _{p_{x}} \frac{\mathcal{E}\left[\log _{2}\left(1+\frac{p_{v} g_{\mathrm{RU}}}{N_{0}+p_{\mathrm{MU}} h_{\mathrm{MR}}}\right)\right\rfloor}{\mathcal{E}\left[p_{v}\right]+P_{\mathrm{c}}} \\
& \text { s.t } \quad \mathrm{C} 1: \mathcal{E}\left[p_{v}\right] \leq P_{\max } \\
& \mathrm{C} 2: \mathcal{P}\left(\log _{2}\left(1+\frac{p_{v} g_{\mathrm{RU}}}{N_{0}+p_{\mathrm{MU}} h_{\mathrm{MR}}}\right)<R_{\min }^{\mathrm{RU}}\right) \leq \sigma_{\mathrm{RU}} \\
& \mathrm{C} 3: \mathcal{P}\left(\log _{2}\left(1+\frac{p_{\mathrm{MU}} g_{\mathrm{MU}}}{N_{0}+p_{v} h_{\mathrm{RM}}}\right)<R_{\min }^{\mathrm{MU}}\right) \leq \sigma_{\mathrm{MU}}
\end{aligned}
$$

where $\mathrm{C} 1$ is the average power constraint of RU. C2 and $\mathrm{C} 3$ are the outage constraints of RU and MU, respectively. 


\section{Energy Efficient Power Allocation Algorithm}

Due to the fractional form of objective function, problem (7) is a non-convex optimization problem and hard to be solved.

Before solving problem (7), we introducing a new problem:

$$
\max _{p_{v} \in \mathbb{Q}} \phi\left(p_{v}, \lambda\right)
$$

where $\mathbb{Q}$ denote the feasible domain defined by constraints $\mathrm{C} 1-\mathrm{C} 3$ in (7) and $\phi\left(p_{v}, \lambda\right)$ is defined as:

$$
\phi\left(p_{v}, \lambda\right)=\left\{\mathcal{E}\left\{\log _{2}\left(1+\frac{p_{v} g_{\mathrm{RU}}}{N_{0}+p_{\mathrm{MU}} h_{\mathrm{MR}}}\right)\right\rfloor-\lambda\left(\mathcal{E}\left[p_{v}\right]+P_{\mathrm{c}}\right)\right\}
$$

Let $\mathcal{S}(\lambda)=\max _{p_{v} \in Q} \phi\left(p_{v}, \lambda\right)$ and $p_{v}(\lambda)=\arg \max _{p_{v} \in Q} \phi\left(p_{v}, \lambda\right)$ be the optimal value and optimal solution of problem (8), respectively.

Theorem 1: The optimal solution $p_{v}^{*}=\underset{p_{v} \in \mathbb{Q}}{\arg \max _{\mathrm{EE}}}$ achieves the optimal value $\lambda^{*}=\max _{p_{x} \in \mathbb{Q}} \xi_{\mathrm{EE}}$ of problem (7), if and only if:

$$
\mathcal{S}\left(\lambda^{*}\right)=0 \text { and } p_{v}\left(\lambda^{*}\right)=p_{v}^{*} .
$$

Proof: Similar proof of Theorem 1 can be found in [10].

Therefore, solving problem (7) is equivalent to find the optimal solution of problem (8) for a given parameter $\lambda$ and then update $\lambda$ until Theorem 1 is fulfilled. By exploiting Dinkelbach method [10], the outer loop algorithm can be summarized in algorithm 1 .

Algorithm 1: Outer Loop of Deriving $\lambda$ in (8)

1:Set tolerance $\vartheta$, initialize $\tau=0$ and $\lambda_{\tau}=0$

2:Solve problem (8) with $\lambda_{\tau}$ to obtain the optimal solution $p_{v}$, i.e., $p_{v, \tau}$

3:while $|\mathcal{S}(\lambda)|>\vartheta$ do

4: $\tau=\tau+1$, update $\lambda_{\tau} \leftarrow \xi_{\mathrm{EE}}$ with $p_{v, \tau-1}$

5: solve (8) with $\lambda_{\tau}$ to obtain $p_{\boldsymbol{x}, \tau}$

6:end while

The convergence of Algorithm 1 has been proved in [10].

In step2, we need to solve problem (8) for a given $\lambda_{\tau}$. In the following, we will discuss how to solve this problem.

Introducing two new functions to express the RU link and CU link outage event:

$$
\begin{gathered}
\delta_{\mathrm{MU}}^{v}\left(p_{v}\right)=\left\{\begin{array}{l}
0, \log _{2}\left(1+\frac{p_{\mathrm{MU}} g_{\mathrm{MU}}}{N_{0}+p_{v} h_{\mathrm{RM}}}\right) \geq R_{\mathrm{min}}^{\mathrm{MU}} \\
1, \log _{2}\left(1+\frac{p_{\mathrm{MU}} g_{\mathrm{MU}}}{N_{0}+p_{v} h_{\mathrm{RM}}}\right)<R_{\mathrm{min}}^{\mathrm{MU}}
\end{array}\right. \\
\delta_{\mathrm{RU}}^{v}\left(p_{v}\right)=\left\{\begin{array}{l}
0, \log _{2}\left(1+\frac{p_{\nu} g_{\mathrm{RU}}}{N_{0}+p_{\mathrm{MU}} h_{\mathrm{MR}}}\right) \geq R_{\min }^{\mathrm{RU}} \\
1, \log _{2}\left(1+\frac{p_{v} g_{\mathrm{RU}}}{N_{0}+p_{\mathrm{MU}} h_{\mathrm{MR}}}\right)<R_{\min }^{\mathrm{RU}}
\end{array}\right.
\end{gathered}
$$

Thus, the RU link and MU link outages constraints can be repressed as:

$$
\begin{aligned}
& \mathcal{E}\left[\delta_{\mathrm{MU}}^{v}\left(p_{v}\right)\right] \leq \sigma_{\mathrm{MU}} \\
& \mathcal{E}\left[\delta_{\mathrm{RU}}^{v}\left(p_{v}\right)\right] \leq \sigma_{\mathrm{RU}}
\end{aligned}
$$

The Lagrangian function of problem (8) is:

$$
\begin{aligned}
\mathfrak{L}\left(p_{v}, \alpha, \beta, \gamma\right)= & \mathcal{E}\left[\log _{2}\left(1+\frac{p_{v} g_{\mathrm{RU}}}{N_{0}+p_{\mathrm{MU}} h_{\mathrm{MR}}}\right)\right\rfloor-\lambda_{\tau}\left\{\mathcal{E}\left[p_{v}\right]+P_{\mathrm{c}}\right\}-\alpha\left\{\mathcal{E}\left[p_{v}\right]-P_{\max }\right\} \\
& -\beta\left\{\mathcal{E}\left[\delta_{\mathrm{MU}}^{v}\left(p_{v}\right)\right]-\sigma_{\mathrm{MU}}\right\}-\gamma\left\{\mathcal{E}\left[\delta_{\mathrm{RU}}^{v}\left(p_{v}\right)\right]-\sigma_{\mathrm{RU}}\right\}
\end{aligned}
$$

where $\alpha, \beta, \gamma$ are the nonnegative dual variables. 
Then, the Lagrange dual function of the primal problem (8) can be written as:

$$
\mathfrak{D}(\alpha, \beta, \gamma)=\max _{p_{v} \geq 0} \mathfrak{L}\left(p_{v}, \alpha, \beta, \gamma\right)
$$

The dual problem is:

$$
\min _{\alpha, \beta, \gamma \geq 0} \mathfrak{D}(\alpha, \beta, \gamma)
$$

Observing that (15) can be rewritten as:

where $\overline{\mathfrak{D}}$ is defined as:

$$
\mathfrak{D}(\alpha, \beta, \gamma)=\mathcal{E}[\overline{\mathfrak{D}}]-\lambda P_{\text {cir }}+\alpha P_{\max }+\beta \sigma_{\mathrm{MU}}+\gamma \sigma_{\mathrm{RU}}
$$

$$
\begin{aligned}
& \quad \overline{\mathfrak{D}}=\max _{p_{v} \geq 0}\left\{\mathcal{F}\left(p_{v}\right)-\beta \delta_{\mathrm{MU}}^{v}\left(p_{v}\right)-\gamma \delta_{\mathrm{RU}}^{v}\left(p_{v}\right)\right\} \\
& \text { with } \mathcal{F}\left(p_{v}\right)=\log _{2}\left(1+\frac{p_{v} g_{\mathrm{RU}}}{N_{0}+p_{\mathrm{MU}} h_{\mathrm{MR}}}\right)-\left(\lambda_{\tau}+\alpha\right) p_{v} . \\
& \text { Define } p_{\mathcal{F}}^{*}=\arg \max \mathcal{F}\left(p_{v}\right), \text { since } \mathcal{F}\left(p_{v}\right) \text { is a concave in } p_{v} \text {, we have: }
\end{aligned}
$$

$$
p_{\mathcal{F}}^{*}=\left(\frac{1}{\left(\lambda_{\tau}+\alpha\right) \ln 2}-\frac{N_{0}+p_{\mathrm{MU}} h_{\mathrm{MR}}}{g_{\mathrm{RU}}}\right)^{+}
$$

where $(x)^{+}=\max \{x, 0\}$. Let $p_{v}^{\mathrm{MU}}$ and $p_{v}^{\mathrm{RU}}$ denote the key value that make the value of $\varepsilon_{\mathrm{MU}}^{v}$ and $\varepsilon_{\mathrm{RU}}^{v}\left(p_{v}\right)$ changes from 0 to 1 , respectively. Thus, we have:

$$
\begin{aligned}
& p_{v}^{\mathrm{MU}}=\frac{p_{\mathrm{MU}} g_{\mathrm{MU}}-N_{0} \gamma_{\min }^{\mathrm{MU}}}{h_{\mathrm{RM}} \gamma_{\min }^{\mathrm{MU}}} \geq 0 \\
& p_{v}^{\mathrm{RU}}=\frac{\gamma_{\min }^{\mathrm{RU}}\left(N_{0}+p_{\mathrm{MU}} h_{\mathrm{MR}}\right)}{g_{\mathrm{RU}}} \geq 0
\end{aligned}
$$

where $\gamma_{\min }^{\mathrm{MU}}=2^{R_{\min }^{\mathrm{MU}}}-1$ and $\gamma_{\min }^{\mathrm{RU}}=2^{R_{\min }^{\mathrm{RU}}}-1$

Therefore, (10) and (11) can be rewritten as

$$
\begin{aligned}
& \delta_{\mathrm{MU}}^{v}\left(p_{v}\right)=\left\{\begin{array}{l}
0, p_{v} \leq p_{v}^{\mathrm{MU}} \\
1, p_{v}>p_{v}^{\mathrm{MU}}
\end{array}\right. \\
& \delta_{\mathrm{RU}}^{v}\left(p_{v}\right)=\left\{\begin{array}{l}
0, p_{v} \geq p_{v}^{\mathrm{RU}} \\
1, p_{v}<p_{v}^{\mathrm{RU}}
\end{array}\right.
\end{aligned}
$$

Let $p_{\kappa}^{*}$ be the optimal solution of problem (18), we have the following Theorem.

Theorem 2: With $p_{\mathcal{F}}^{*}, p_{v}^{\mathrm{MU}}$ and $p_{v}^{\mathrm{RU}}$, the optimal solution of (18) is given by the following cases: case 1: if $p_{v}^{\mathrm{MU}}<p_{\mathcal{F}}^{*}<p_{v}^{\mathrm{RU}}$, then we have:

$$
p_{v}^{*}=\left\{\begin{array}{l}
p_{v}^{\mathrm{MU}}, \mathcal{F}\left(p_{v}^{\mathrm{MU}}\right)-\gamma>\max \left\{\mathcal{F}\left(p_{\mathcal{F}}^{*}\right)-\beta-\gamma, \mathcal{F}\left(p_{v}^{\mathrm{RU}}\right)-\beta\right\} \\
p_{\mathcal{F}}^{*}, \mathcal{F}\left(p_{\mathcal{F}}^{*}\right)-\beta-\gamma>\max \left\{\mathcal{F}\left(p_{v}^{\mathrm{MU}}\right)-\gamma, \mathcal{F}\left(p_{v}^{\mathrm{RU}}\right)-\beta\right\} \\
p_{v}^{\mathrm{RU}}, \mathcal{F}\left(p_{v}^{\mathrm{RU}}\right)-\beta>\max \left\{\mathcal{F}\left(p_{v}^{\mathrm{MU}}\right)-\gamma, \mathcal{F}\left(p_{\mathcal{F}}^{*}\right)-\beta-\gamma\right\}
\end{array}\right.
$$

case 2: if $p_{v}^{\mathrm{RU}} \leq p_{\mathcal{F}}^{*} \leq p_{v}^{\mathrm{MU}}$, then we have: $p_{v}^{*}=p_{\mathcal{F}}^{*}$.

case 3: if $p_{\mathcal{F}}^{*} \leq p_{v}^{\mathrm{MU}}<p_{v}^{\mathrm{RU}}$, then we have: $p_{v}^{*}=\left\{\begin{array}{l}p_{\mathcal{F}}^{*}, \mathcal{F}\left(p_{\mathcal{F}}^{*}\right)-\gamma>\mathcal{F}\left(p_{v}^{\mathrm{RU}}\right)-\beta \\ p_{v}^{\mathrm{RU}}, \mathcal{F}\left(p_{v}^{\mathrm{RU}}\right)-\beta>\mathcal{F}\left(p_{\mathcal{F}}^{*}\right)-\gamma\end{array}\right.$.

case 4: if $p_{\mathcal{F}}^{*}<p_{v}^{\mathrm{RU}}<p_{v}^{\mathrm{MU}}$, then we have: $p_{v}^{*}=\left\{\begin{array}{l}p_{\mathcal{F}}^{*}, \mathcal{F}\left(p_{\mathcal{F}}^{*}\right)-\gamma>\mathcal{F}\left(p_{v}^{\mathrm{RU}}\right) \\ p_{v}^{\mathrm{RU}}, \mathcal{F}\left(p_{v}^{\mathrm{RU}}\right)>\mathcal{F}\left(p_{\mathcal{F}}^{*}\right)-\gamma\end{array}\right.$.

case 5: if $p_{v}^{\mathrm{MU}}<p_{v}^{\mathrm{RU}} \leq p_{\mathcal{F}}^{*}$, then we have: $p_{v}^{*}=\left\{\begin{array}{l}p_{v}^{\mathrm{MU}}, \mathcal{F}\left(p_{v}^{\mathrm{MU}}\right)-\gamma>\mathcal{F}\left(p_{\mathcal{F}}^{*}\right)-\beta \\ p_{\mathcal{F}}^{*}, \mathcal{F}\left(p_{\mathcal{F}}^{*}\right)-\beta>\mathcal{F}\left(p_{v}^{\mathrm{MU}}\right)-\gamma\end{array}\right.$.

case 6: if $p_{v}^{\mathrm{RU}}<p_{v}^{\mathrm{MU}}<p_{\mathrm{g}}^{*}$, then we have: $p_{v}^{*}=\left\{\begin{array}{l}p_{v}^{\mathrm{MU}}, \mathcal{F}\left(p_{v}^{\mathrm{MU}}\right)>\mathcal{F}\left(p_{\mathcal{F}}^{*}\right)-\beta \\ p_{\mathcal{F}}^{*}, \mathcal{F}\left(p_{\mathcal{F}}^{*}\right)-\beta>\mathcal{F}\left(p_{v}^{\mathrm{MU}}\right)\end{array}\right.$.

\section{Proof:}

Combing with formula (16) and concave function $\mathcal{F}\left(p_{v}\right)$, for $p_{v}^{\mathrm{MU}}<p_{v}^{\mathrm{RU}}$, we have: 


$$
\mathcal{F}\left(p_{v}\right)-\beta \varepsilon_{v}^{\mathrm{MU}}\left(p_{v}\right)-\gamma \varepsilon_{v}^{\mathrm{RU}}\left(p_{v}\right)=\left\{\begin{array}{l}
\mathcal{F}\left(p_{v}\right)-\gamma, p_{v} \leq p_{v}^{\mathrm{MU}} \\
\mathcal{F}\left(p_{v}\right)-\beta-\gamma, p_{v}^{\mathrm{MU}}<p_{v}<p_{v}^{\mathrm{RU}} \\
\mathcal{F}\left(p_{v}\right)-\beta, p_{v} \geq p_{v}^{\mathrm{RU}}
\end{array}\right.
$$

For $p_{v}^{\mathrm{RU}}<p_{v}^{\mathrm{MU}}$, we have:

$$
\mathcal{F}\left(p_{v}\right)-\beta \varepsilon_{v}^{\mathrm{MU}}\left(p_{v}\right)-\gamma \varepsilon_{v}^{\mathrm{RU}}\left(p_{v}\right)=\left\{\begin{array}{l}
\mathcal{F}\left(p_{v}\right)-\gamma, p_{v}<p_{v}^{\mathrm{RU}} \\
\mathcal{F}\left(p_{v}\right), p_{v}^{\mathrm{RU}} \leq p_{v} \leq p_{v}^{\mathrm{MU}} \\
\mathcal{F}\left(p_{v}\right)-\beta, p_{v}>p_{v}^{\mathrm{MU}}
\end{array}\right.
$$

Based on (24) and (25), take $p_{\mathfrak{g}}^{*}$ into consideration, we have six different forms of the objective function $\mathcal{F}\left(p_{v}\right)-\beta \varepsilon_{\mathrm{MU}}^{v}\left(p_{v}\right)-\gamma \mathcal{E}_{\mathrm{RU}}^{v}\left(p_{v}\right)$ as shown in Fig.1-Fig.6. Note: In the figures, the dotted line denotes function $\mathcal{F}\left(p_{v}\right)$ and the solid line denotes function $\mathcal{F}\left(p_{v}\right)-\beta \delta_{\mathrm{MU}}^{v}\left(p_{v}\right)-\gamma \delta_{\mathrm{RU}}^{v}\left(p_{v}\right)$.

case 1: In Fig. $1, y_{1}=\mathcal{F}\left(p_{v}^{\mathrm{MU}}\right)-\gamma, y_{2}=\mathcal{F}\left(p_{\mathrm{g}}^{*}\right)-\beta-\gamma, y_{3}=\mathcal{F}\left(p_{v}^{\mathrm{RU}}\right)-\beta$. The optimal $p_{v}^{*}$ rely on $y_{1}, y_{2}, y_{3}$. case 2: In Fig.2, $y_{1}=\mathcal{F}\left(p_{v}^{\mathrm{RU}}\right), y_{2}=\mathcal{F}\left(p_{\mathfrak{g}}^{*}\right), y_{3}=\mathcal{F}\left(p_{v}^{\mathrm{MU}}\right)$. Since $\mathcal{F}\left(p_{\kappa}\right)$ is a concave function, thus $p_{v}^{*}=p_{\mathfrak{g}}^{*}$ case 3: In Fig.3, $y_{1}=\mathcal{F}\left(p_{\mathfrak{g}}^{*}\right)-\gamma, y_{2}=\mathcal{F}\left(p_{v}^{\mathrm{MU}}\right)-\gamma, y_{3}=\mathcal{F}\left(p_{v}^{\mathrm{RU}}\right)-\beta$. Since $y_{1}>y_{2}$, the optimal $p_{v}^{*}$ rely on $y_{1}, y_{3}$. case 4: In Fig.4, $y_{1}=\mathcal{F}\left(p_{\mathfrak{g}}^{*}\right)-\gamma, y_{2}=\mathcal{F}\left(p_{v}^{\mathrm{RU}}\right), y_{3}=\mathcal{F}\left(p_{v}^{\mathrm{MU}}\right)$. Since $y_{2}>y_{1}$, the optimal $p_{v}^{*}$ rely on $y_{1}, y_{2}$. case 5: In Fig.5, $y_{1}=\mathcal{F}\left(p_{v}^{\mathrm{MU}}\right)-\gamma, y_{2}=\mathcal{F}\left(p_{v}^{\mathrm{RU}}\right)-\beta, y_{3}=\mathcal{F}\left(p_{\mathfrak{g}}^{*}\right)-\beta$. Since $y_{3}>y_{2}$, the optimal $p_{v}^{*}$ rely on $y_{1}, y_{3}$.

case 6: In Fig.6, $y_{1}=\mathcal{F}\left(p_{v}^{\mathrm{RU}}\right), y_{2}=\mathcal{F}\left(p_{v}^{\mathrm{MU}}\right), y_{3}=\mathcal{F}\left(p_{\mathcal{F}}^{*}\right)-\beta$. Since $y_{2}>y_{1}$, the optimal $p_{v}^{*}$ rely on $y_{2}, y_{3}$.

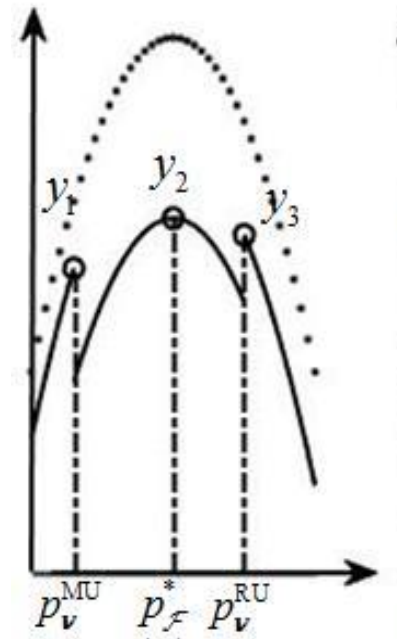

(a)

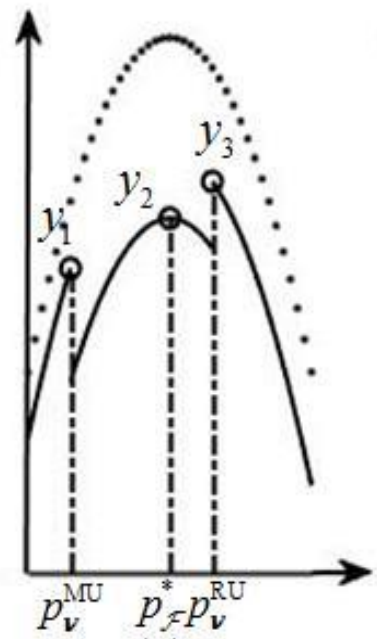

(b)

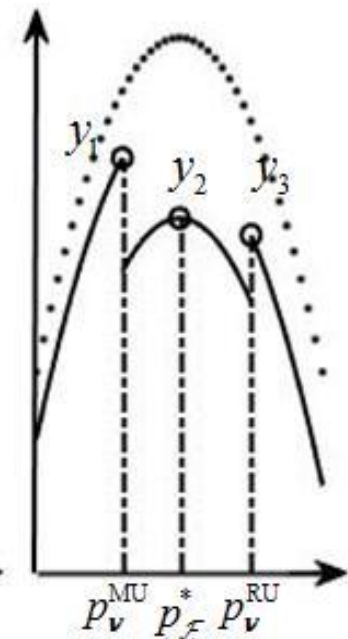

(c)

Fig. 1: Case 1.

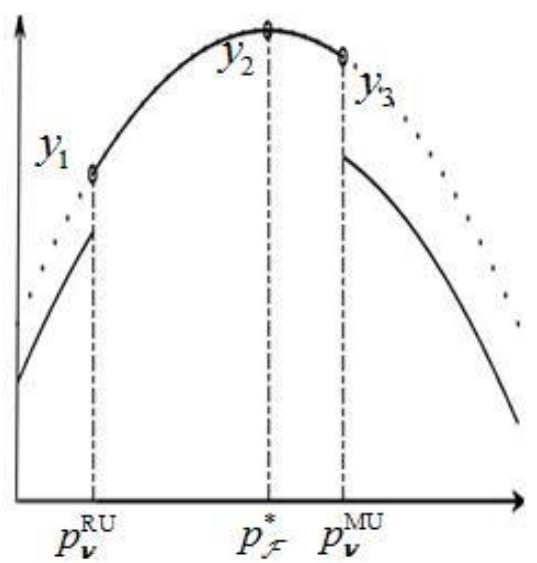

Fig. 2: Case 2. 


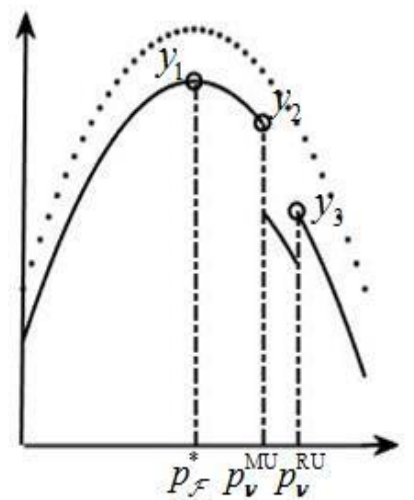

(a)

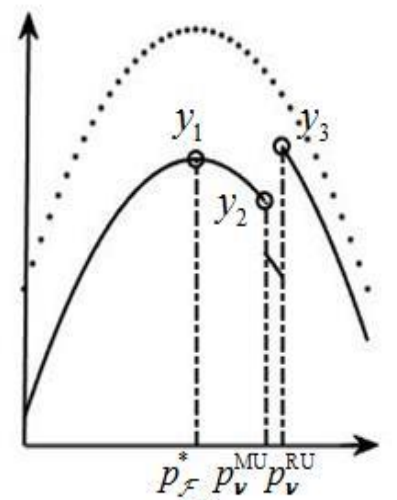

(b)

Fig. 3: Case 3.

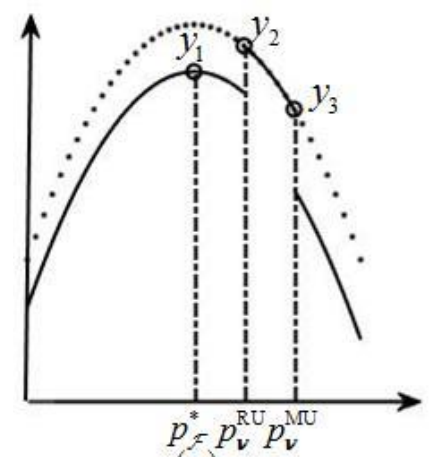

(a)

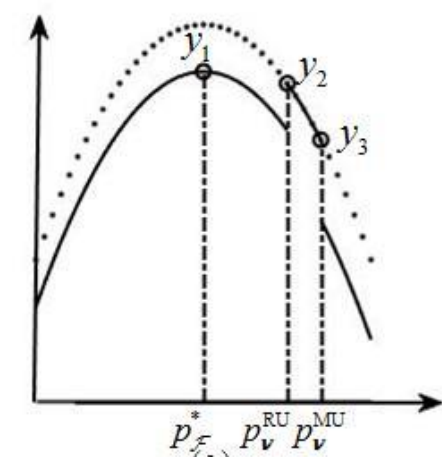

(b)

Fig. 4: Case 4.
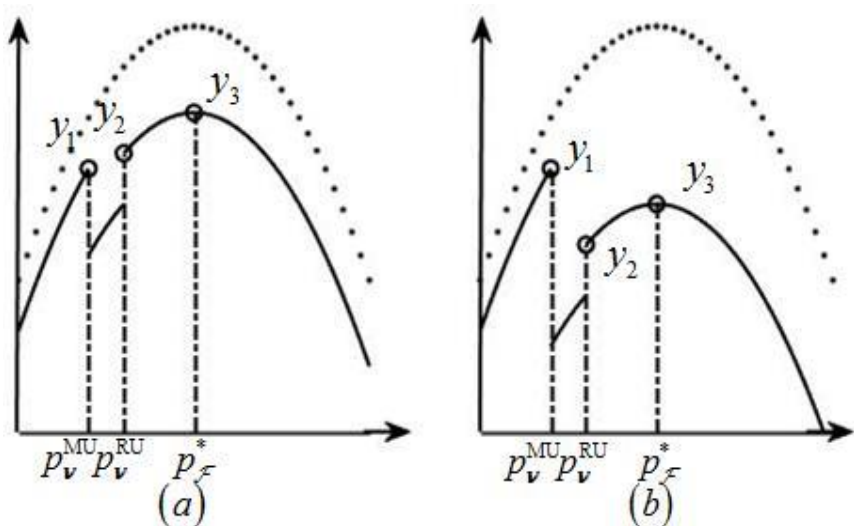

Fig. 5: Case 5.

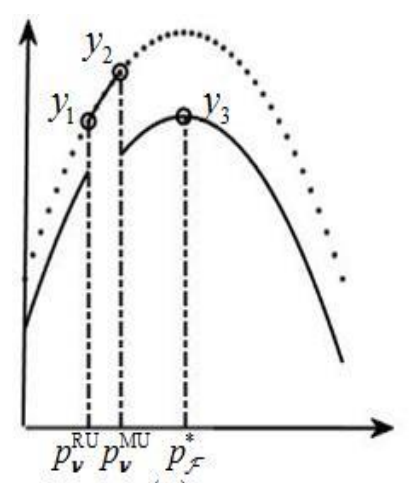

(a)

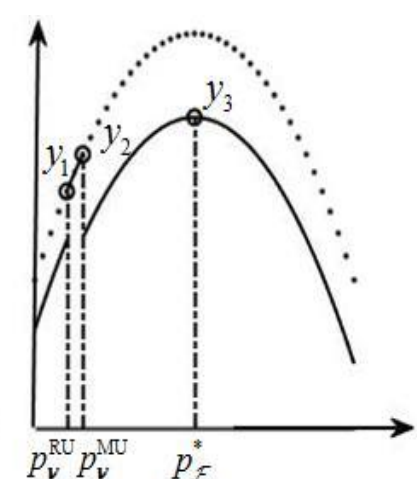

(b)

Fig. 6: Case 6. 
Here, we use subgradient method [11] to obtain the optimal Lagrange multipliers:

$$
\begin{aligned}
& \alpha_{t+1}=\left[\alpha_{t}-\eta_{t}^{\alpha}\left(\mathcal{E}\left[p_{v}\right]-P_{\max }\right)\right]^{+} \\
& \beta_{t+1}=\left[\beta_{t}-\eta_{t}^{\beta}\left\{\mathcal{E}\left[\delta_{\mathrm{MU}}^{v}\left(p_{v}\right)\right]-\sigma_{\mathrm{MU}}\right\}\right]^{+} \\
& \gamma_{t+1}=\left[\gamma_{t}-\eta_{t}^{\gamma}\left\{\mathcal{E}\left[\varepsilon_{\mathrm{RU}}^{v}\left(p_{v}\right)\right]-\sigma_{\mathrm{RU}}\right\}\right]^{+}
\end{aligned}
$$

where $\eta_{t}^{\alpha}, \eta_{t}^{\beta}, \eta_{t}^{\gamma}$ are small positive step sizes for the $t$-th iteration. The subgradient updates of (26), (27), (28) are guaranteed to converge to the optimal $\alpha, \beta, \gamma$ as long as $\eta_{t}^{\alpha}, \eta_{t}^{\beta}, \eta_{t}^{\gamma}$ are chosen to be sufficiently small [11].

Algorithm2: Inner loop of solving (8) with given $\lambda_{\tau}$ based on sub-gradient method

1: Initialization $t=0, \alpha_{t}, \beta_{t}, \gamma_{t}$, calculate $p_{v}^{\mathrm{MU}}$ and $p_{v}^{\mathrm{D}}$ according to (20) and (21), respectively.

2: calculate $p_{\mathrm{g}}^{*}$ according to (19), then calculate $p_{v}^{*}$ according to Theorem 2 .

3: $t=t+1$, update $\alpha_{t+1}, \beta_{t+1}, \gamma_{t+1}$ according to (26), (27), (28).

4: if the multipliers $\alpha, \beta, \gamma$ are convergent, return and stop the algorithm2; otherwise go to step2

\section{Simulation}

In this section, some numerical results are presented to evaluate the performance of the proposed schemes. All the channels are assumed to be Rayleigh-fading, the channel power gains are exponentially distributed with unit mean for $g_{\mathrm{MU}}$ and $g_{\mathrm{RU}}$, and 0.5 mean for $h_{\mathrm{RM}}$ and $h_{\mathrm{MR}}$. Without loss of generality, we set $\sigma_{\mathrm{MU}}=\sigma_{\mathrm{RU}}=\sigma, N_{0}=10^{-5} \mathrm{~mW}, p_{\mathrm{MU}}=200 \mathrm{~mW}, R_{\min }^{\mathrm{MU}}=R_{\min }^{\mathrm{RU}}=2 \mathrm{bit} / \mathrm{s} / \mathrm{Hz}, P_{\mathrm{c}}=10 \mathrm{~mW}$.

Fig.7 illustrates the evolution of the proposed algorithm with different $P_{\max }$ under $\sigma=0.2$. Note, the proposed algorithm consists of two loops, we only consider the effect of outer loop iterations $\tau$. It is observed that the algorithm converge to the optimal $\lambda$ fast.

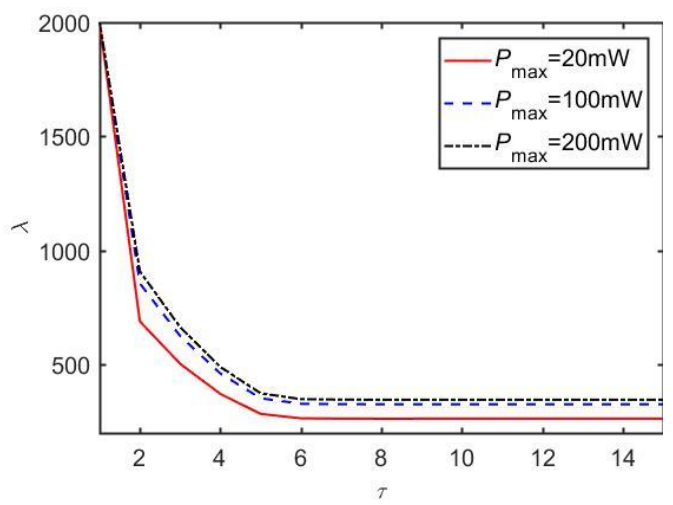

Fig. 7: Convergence evolution of the proposed algorithm.

To emphasize the advantages of the proposed scheme, we introduce a new power allocation problem:

$$
\begin{aligned}
& \text { OP2 } \max _{p_{v}} \mathcal{E}\left[\log _{2}\left(1+\frac{p_{v} g_{\mathrm{RU}}}{N_{0}+p_{\mathrm{MU}} h_{\mathrm{MR}}}\right)\right\rfloor \\
& \text { s.t } \quad \mathrm{C}: \mathcal{E}\left[p_{v}\right] \leq P_{\max } \\
& \quad \mathrm{C} 2: \mathcal{P}\left(\log _{2}\left(1+\frac{p_{v} g_{\mathrm{RU}}}{N_{0}+p_{\mathrm{MU}} h_{\mathrm{MR}}}\right)<R_{\min }^{\mathrm{RU}}\right) \leq \sigma_{\mathrm{RU}} \\
& \quad \mathrm{C} 3: \mathcal{P}\left(\log _{2}\left(1+\frac{p_{\mathrm{MU}} g_{\mathrm{MU}}}{N_{0}+p_{v} h_{\mathrm{RM}}}\right)<R_{\min }^{\mathrm{MU}}\right) \leq \sigma_{\mathrm{MU}}
\end{aligned}
$$

Then problem OP2 becomes the traditional ergodic rate maximization problem. Similar to problem (8), we can also use algorithm 2 to solve problem OP2 with appropriate medications. We name the scheme to solve the ergodic rate maximization problem as ERMP algorithm. Next, we will compare the proposed algorithm with the ERPA. As shown in Fig.8, the proposed algorithm has a higher energy efficiency than ERMP algorithm. This is because the proposed algorithm always obtains the optimal power allocation that achieves the highest energy efficiency. 
As shown in Fig.9, the energy efficiency of the both algorithms increase with the increasing of $\sigma$. This is because small $\sigma$ indicates strict outage constraint, large $\sigma$ indicates loose outage constraint. In addition, the proposed algorithm has better performance than ERMP algorithm in improving energy efficiency.

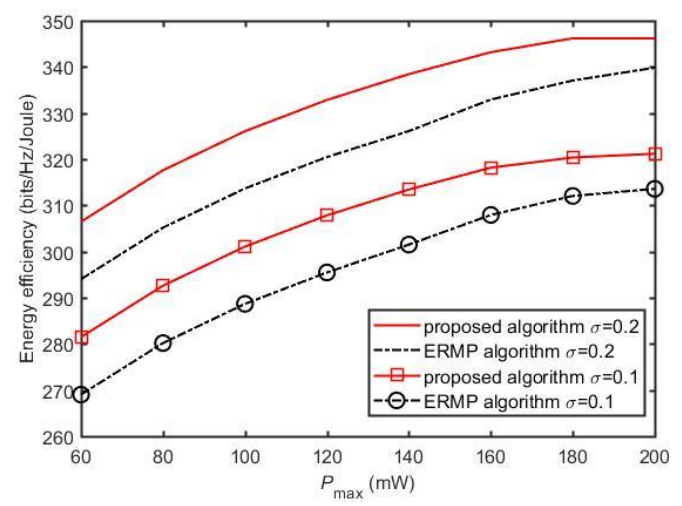

Fig. 8: Energy efficiency versus average transmit power threshold.

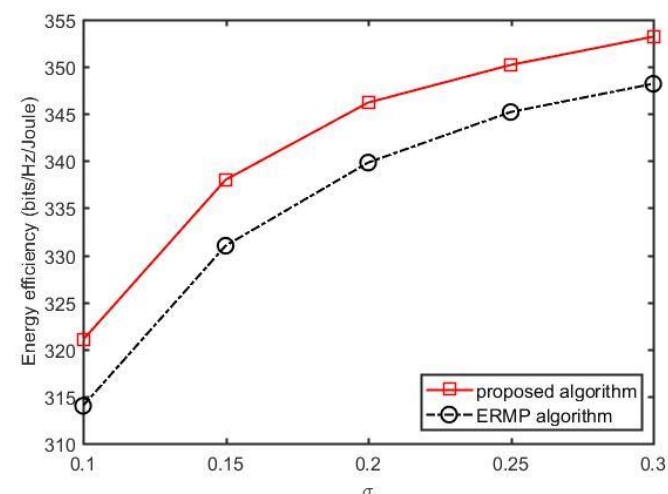

Fig. 9: Energy efficiency versus maximum outage probability.

\section{Conclusions}

Most of the previous works consider resource allocation of HC-RAN with perfect CSI. In fact, it is difficult and costly to acquire accurate CSI. In this paper, we investigate the energy efficient power allocation of HC-RAN with partial CSI. An efficient iterative power allocation scheme has been derived to maximize the averaged energy efficiency of HC-RAN.

\section{Acknowledgements}

This paper is supported by Postdoctoral fund of Jiangsu Province (SBH17024), Jiangsu University of Natural Science Foundation (15KJB510026), Natural Science Foundation of Jiangsu Province (BK20150866), Introduction of Talent Fund of Nanjing University of Posts and Telecommunications(NY215046, NY217056). National Natural Science Fund (61701255).

\section{References}

[1] Y.Z Li, T. Jiang, K. Luo, et.al. Green heterogeneous cloud radio access networks: potential techniques, performance trade-offs, and challenges. IEEE Commun. Mag. 2017, 55(11):33-39.

[2] A. Ismail and S. Basem. Sophisticated online learning scheme for green resource allocation in $5 \mathrm{G}$ heterogeneous cloud radio access networks. IEEE Trans. Mobile Comput. 2018, PP(99):1-16.

[3] M. G. Peng, Y. Y. Wang, T. Dang, et.al. Cost-efficient resource allocation in cloud radio access networks with heterogeneous fronthual expenditures. IEEE Trans. Wirel. Commun. 2017, 16(7):4626-4638.

[4] A Mudassar, R. Quratulain, N. Muhammad, et. al. Joint user association, power allocation, and throughput maximization in 5G H-CRAN networks. IEEE Trans. Veh. Technol. 2017, 66(10):9254-9262.

[5] Q. T. Vien, T. A. Le, B. Barn, et al. Optimizing energy efficiency of non-orthogonal multiple access for wireless 
backhaul in heterogeneous cloud radio access network. IET Commun. 2016, 10(8):2516-2524.

[6] J. C. Liu, S. Zhou, J. Gong, et al. Statistical multiplexing gain analysis of heterogeneous virtual base station pools in cloud radio access networks. IEEE Trans. Wirel. Commun. 2016, 15(8):5681-5694.

[7] M. G. Peng, K. C. Zhang, J. M. Jiang, et. al. Energy-efficient resource assignment and power allocation in heterogeneous cloud radio access networks. IEEE Trans. Veh. Technol. 2015, 64(11):5275-5287.

[8] J. Li, M. G. Peng, Y. L. Yu, et.al. Energy-efficient joint congestion control and resource optimization in heterogeneous cloud radio access networks. IEEE Trans. Veh. Technol. 2016, 65(12):9873-9887.

[9] Y. Zhang, Y. Wang, and B. Fan. SDN based optimal user cooperation and energy efficient resource allocation in cloud assisted heterogeneous networks. IEEE Access. 2017, 5:1469-1481.

[10] W. Dinkelbach. On nonlinear fractional programming. Management Science. 1967, 13:492-498.

[11] S. Boyd, L. Xiao and A. Mutapcic. Subgradient Methods. http://www.stanford.edu./class/ee392o/subgrad_method.pdf 